\title{
Age is the main determinant of glycated hemoglobin levels in a general Polish population without diabetes: The NATPOL 2011 Study
}

\author{
Bartosz Symonides ${ }^{1, B-F}$, Bogdan Solnica ${ }^{2, A, C, E, F}$, Grzegorz Placha ${ }^{1, B, E, F}$, Ewa Pędzich-Placha, ${ }^{1, B, E, F}$, \\ Marcin Rutkowski3 ${ }^{3, E, E, F}$, Piotr Bandosz ${ }^{3, B, E, F}$, Zbigniew Gaciong ${ }^{1, A, E, F}$, Tomasz Zdrojewski, ${ }^{3, A, E}$ \\ ${ }^{1}$ Department of Internal Medicine, Hypertension and Vascular Disease, Medical University of Warsaw, Poland \\ 2 Department of Diagnostics, Chair of Clinical Biochemistry, Jagiellonian University Medical College, Kraków, Poland \\ ${ }^{3}$ Department of Hypertension and Diabetology, Medical University of Gdańsk, Poland \\ A - research concept and design; $\mathrm{B}$ - collection and/or assembly of data; C - data analysis and interpretation; \\ $D$ - writing the article; $E$ - critical revision of the article; $F$ - final approval of the article
}

Address for correspondence

Bartosz Symonides

E-mail: bartosz.symonides@wum.edu.pl

\section{Funding sources}

The NATPOL 2011 Study was partially funded by the Polish Ministry of Health as a publicly funded project representing part of the National Cardiovascular Disease Prevention and Treatment Programme and with statutory grants from the Medical University of Gdańsk and the Medical University of Warsaw. It was also partly funded by the following industry sponsors. The main sponsor of the project: Sanofi-Aventis - unrestricted educational grant; Abbott Laboratories Poland Ltd - sponsor with unrestricted educational grant; Siemens Ltd - partner of the project - unrestricted educational grant; Polpharma - partner of the project - unrestricted educational grant - in the part of the project dedicated to heart failure. The funding agencies had no involvement in the design or conduct of the study, collection, management, analysis, and interpretation of data, or drafting the manuscript.

\section{Conflict of interest}

None declared

Received on July 27, 2016

Reviewed on 0ctober 24, 2016

Accepted on May 29, 2018

Published online on January 24, 2019

Cite as

Symonides B, Solnica B, Placha G. Age is the main determinant of glycated hemoglobin levels in a general Polish population without diabetes: The NATPOL 2011 Study. Adv Clin Exp Med. 2019;28(5):659-664. doi:10.17219/acem/91790

DOI

10.17219/acem/91790

\section{Copyright}

Copyright by Author(s)

This is an article distributed under the terms of the Creative Commons Attribution Non-Commercial License (http://creativecommons.org/licenses/by-nc-nd/4.0/)

\begin{abstract}
Background. Measurements of glycated hemoglobin (HbA1c) in non-diabetics can identify subjects who are at increased risk for future cardiovascular (CV) events. There is no consensus agreement whether the addition of HbA1c improves the $\mathrm{CV}$ risk prediction.

Objectives. The objective of this study was to assess mean values of HbA1c levels in a representative sample of general, diabetes mellitus (DM)-free Polish population, and its subgroups, and to identify important covariants.

Material and methods. HbA1c was measured in blood samples collected from 1,868 participants (males/ females (M/F) 901/967, age: range 18-74, mean 44.03 years) of NATPOLL 2011 study without previously and newly diagnosed DM. Univariate and multivariate analyses of $\mathrm{HbATc}$ level in relationship to age, body mass index (BMI), waist circumference (WC), systolic blood pressure (SBP), diastolic blood pressure (DBP), fasting plasma glucose (FPG), lipids, creatinine, (-reactive protein (CRP), gender, and smoking status were performed.

Results. Mean HbA1c level was $5.46 \pm 0.31 \%$ in the entire population and significantly higher levels were found in subjects with male gender, hypertension, fasting hyperglycemia, abdominal obesity, and higher BMI values but not in smokers. Univariate analysis revealed numerous significant correlations of $\mathrm{HbAl}$ with the highest values correlation coefficient values for age $(r=0.55), F P G(r=0.43), W C(r=0.36)$, and BMI $(r=0.36)$. The best, final multivariate model explained $40 \%$ of HbA1c variance and the most important covariant was the age, explaining approx. 50\% of R2, followed by FPG and BMI.
\end{abstract}

Conclusions. HbA1c in non-diabetic level is associated with certain CV risk factors, mainly with age. Since known risk factors explain less than a half of HbAlc variance, the inclusion of HbA1c into the assessment may increase the performance of algorithms predicting $C V$ risk.

Key words: glycated hemoglobin, age, lipids 
According to different algorithms for cardiovascular (CV) risk, type 2 diabetes mellitus (DM2) is considered an equivalent of coronary heart disease (CHD). Also, patients with diabetes, when compared to non-diabetic subjects, have a higher prevalence of $\mathrm{CHD}$, a greater extent of coronary ischemia, and are more likely to have a myocardial infarction (MI) and silent myocardial ischemia.

In the general population, there is even a larger group of subjects who do not meet the diagnostic criteria for diabetes but are affected by different degrees of dysglycemia, including impaired fasting glucose or impaired glucose tolerance. Measurements of glycated hemoglobin (HbA1c) can identify subjects who are at increased risk not only for the future development of DM2,1,2 but also for CV events. $^{3-6}$

In a meta-analysis, HbA1c was found to be the best marker of dysglycemia and CV risk in subjects without DM2. ${ }^{7}$ In the INTERHEART study, the association between HbA1c and the history of MI was stronger in non-diabetics than in patients with DM2. ${ }^{8}$ Mechanisms underlying this association remain unclear and there is no consensus agreement whether addition of HbA1c level as a diagnostic tool improves the risk prediction for cardiovascular disease (CVD). ${ }^{9}$

\section{Material and methods}

A detailed design of the NATPOL 2011 Survey has been reported elsewhere. ${ }^{10}$ Briefly, the NATPOL 2011 survey was designed as a cross-sectional representative observational study to assess the prevalence of main atherosclerotic CVD risk factors in Poland. It was carried out on a representative sample of Polish residents aged 18-79 years. The participants were randomly selected in bundles, in a stratified, proportional draw performed in 3 stages. The response rate among respondents who were invited and eligible for the study was equal to $66.4 \%$. Finally, 2,413 subjects (1,245 females and 1,168 males) participated in the survey. Subjects with previously diagnosed diabetes, with HbA1c level $>6.5 \%$ and incomplete biochemical measurements were excluded from the current analysis. Therefore, the final analysis was performed in $\mathrm{n}=1,868$ subjects (male/ female $901 / 967$, mean age $44.03 \pm 16.28$ years) and the comparisons between the subgroups with and without the following: hypertension, fasting hyperglycaemia, abdominal obesity and according to body mass index (BMI) and current smoking status were performed.

The Institutional Ethics Committee at the Medical University in Gdańsk (Poland) approved the study protocol; all participants provided written informed consent.

The examination was performed by 234 well-trained nurses who lived in or close to the randomly selected geographical bundles. Participants were examined during 2 visits at subjects' homes. The examination of an individual subject comprised the following components: completing the questionnaire, taking blood pressure readings and anthropometric measurements (weight, height, waist circumference (WC)), and collecting blood and urine samples.

The questionnaire was completed during the $1^{\text {st }}$ visit. Only selected items of the questionnaire were used for the following substudy including age, history of diabetes and hypertension, antihypertensive and statin use, and smoking.

Blood pressure readings were taken 3 times during the $1^{\text {st }}$ and the $2^{\text {nd }}$ visit using fully automated oscillometric blood pressure measuring device (A\&D UA 767 A\&D Company, Tokyo, Japan). Mean values of $2^{\text {nd }}$ and $3^{\text {rd }}$ measurements from 2 visits were used for the analysis. Hypertension was diagnosed if during both visits mean systolic blood pressure (SBP) was $\geq 140 \mathrm{~mm} \mathrm{Hg}$ and/or mean diastolic blood pressure (DBP) was $\geq 90 \mathrm{~mm} \mathrm{Hg}$ or if the patient was taking hypertensive drugs over the past 2 weeks due to an earlier diagnosis of hypertension.

Anthropometric measurements used in the following substudy included weight, height and WC. Weight was measured with the subject shoeless and dressed in light clothes (without outer garments - jackets, coats, etc.), using approved personal electronic scales, with accuracy to the nearest $0.1 \mathrm{~kg}$. Height was measured using a portable personal measuring device with accuracy to the nearest centimeter, and WC using a tailor's tape measure, with an accuracy to the nearest $0.5 \mathrm{~cm}$. Overweight was defined as BMI $25.0-29.9 \mathrm{~kg} / \mathrm{m}^{2}$, obesity as BMI $\geq 30 \mathrm{~kg} / \mathrm{m}^{2}$ and abdominal obesity as $\mathrm{WC} \geq 102 \mathrm{~cm}$ in men and $\mathrm{WC} \geq 88 \mathrm{~cm}$ in women.

Blood and urine samples were taken from subjects at the $2^{\text {nd }}$ visit, after 10 . to 12 -hour fasting. Frozen plasma and serum samples were transported to the central laboratory, where blood and urine analyses were carried out.

Routine blood tests: fasting plasma glucose (FPG), total cholesterol (TC), high-density lipoprotein cholesterol (HDL-C), serum triglycerides (TG), plasma creatinine, serum $C$-reactive protein (CRP), and urine albumin and creatinine were measured on the Architect c8000 chemistry analyzer (Abbott Laboratories, Chicago, USA). Glucose was measured using the hexokinase method. Serum cholesterol was measured with the enzymatic method, using cholesterol esterase and cholesterol oxidase; serum HDL-C was measured with the direct method using Accelerator Selective Detergent (Abbott Laboratories, Chicago, USA) with accelerated non-HDL-C oxidation and HDL-C dissolving. Serum triglycerides were measured by the enzymatic method using glycerol kinase and glycerol phosphate oxidase. Low-density lipoprotein cholesterol (LDL-C) was calculated using Friedewald formula. If TG concentration was $>350 \mathrm{mg} / \mathrm{dL}$, the Friedewald formula was not used.

Hypercholesterolemia was defined as TC $\geq 190 \mathrm{mg} / \mathrm{dL}$ ( $4.9 \mathrm{mmol} / \mathrm{L})$ or taking statins. Fasting hypergylcemia was defined as fasting glucose $\geq 100 \mathrm{mg} / \mathrm{dL}$ ( $\geq 5.6 \mathrm{mmol} / \mathrm{L})$. Plasma creatinine was measured using Jaffe method, method and CRP in serum using immunoturbidimetric method. 
Serum insulin was measured on the Architect i2000sr, immunochemistry analyzer (Abbott Laboratories) using chemiluminescence immunoassay. Urine albumin was measured by the immunoturbidimetric method and creatinine using the Jaffe method. Albumin-creatinine ratio (ACR) was calculated on the basis of urine albumin and urine creatinine measurement.

HbA1c level was measured in ethylenediaminetetraacetic acid (EDTA) whole blood samples using turbidimetric inhibition immunoassay on the Cobas Integra 800 analyzer (Roche Diagnostics, Mannheim, Germany). The statistical analysis of the data was performed using $\mathrm{R}$ package v. 3.0.2 (The R Foundation, Vienna, Austria).

We compared mean values of HbA1c in both genders, in subjects with and without the following: hypertension, impaired fasting glucose and current smoking status. Due to the skewness, ACR values were log-transformed. We used the student's t-test or analysis of variance
(ANOVA) and $\chi^{2}$ test, where appropriate. All results were presented as means \pm standard deviation (SD). Significant $\mathrm{p}$-value was set at $\mathrm{p}<0.05$.

We performed univariate analysis calculating correlation coefficients of $\mathrm{HbA1c}$ with the following quantitative parameters: age, BMI, WC, SBP, DBP, FPG, insulin, lipids, creatinine, CRP, and ACR using Pearson's method.

For the multivariate analysis, we included all quantitative parameters mentioned above, and additionally gender and smoking habit, removing variables that highly correlated with the others $(r>0.6)$ namely DBP, WC and LDL-C. The selection of variables for the final model was performed using "leaps" R package applying all subsets regression method. We calculated standardized beta coefficients "QuantPsyc" R package) together with corresponding significance and $\mathrm{R}^{2}$ for all variables selected for the final model using the "relaimpo" R package with "lmg", "last", "first", and "Pratt" algorithms.

Table 1. Characteristics of all participants and men vs women

\begin{tabular}{|c|c|c|c|c|}
\hline \multirow{2}{*}{ Parameter } & All subjects & Men & Women & \multirow{2}{*}{$p$-value } \\
\hline & mean \pm SD or $n[\%]$ & mean \pm SD or $n[\%]$ & mean \pm SD or $n[\%]$ & \\
\hline Number of patients & 1868 & $901(48.2)$ & $967(51.8)$ & - \\
\hline Age [years] & $44.03 \pm 16.28$ & $43.34 \pm 15.66$ & $44.69 \pm 16.82$ & 0.0725 \\
\hline BMI $\left[\mathrm{kg} / \mathrm{m}^{2}\right]$ & $26.2 \pm 4.8$ & $26.9 \pm 4.5$ & $25.5 \pm 5.0$ & $<0.0001$ \\
\hline Normal/low BMI & $810(43.4)$ & 303 (33.6) & $507(52.4)$ & $<0.0001$ \\
\hline Overweight & $687(36.8)$ & $394(43.7)$ & $293(30.3)$ & $<0.0001$ \\
\hline Obesity & $371(19.9)$ & $204(22.6)$ & $167(17.3)$ & $<0.0001$ \\
\hline$W C[\mathrm{~cm}]$ & $90.35 \pm 13.92$ & $96.5 \pm 12.22$ & $84.62 \pm 12.93$ & $<0.0001$ \\
\hline Abdominal obesity, $n$ & $1104(59.1)$ & $537(59.6)$ & $567(58.6)$ & 0.7062 \\
\hline $\mathrm{SBP}[\mathrm{mm} \mathrm{Hg}]$ & $127.1 \pm 17.8$ & $131.5 \pm 16.6$ & $123.1 \pm 17.9$ & $<0.0001$ \\
\hline $\mathrm{DBP}[\mathrm{mm} \mathrm{Hg}]$ & $79.8 \pm 9.9$ & $80.9 \pm 10.3$ & $78.8 \pm 9.5$ & $<0.0001$ \\
\hline Hypertension, n & $551(29.5)$ & $299(33.2)$ & $252(26.1)$ & $<0.0001$ \\
\hline Glucose [mg/dL] & $90.69 \pm 11.81$ & $92.83 \pm 11.94$ & $88.69 \pm 11.33$ & $<0.0001$ \\
\hline Glucose [mmol/L] & $5.04 \pm 0.66$ & $5.16 \pm 0.66$ & $4.93 \pm 0.63$ & $<0.0001$ \\
\hline Insulin [mLU/L] & $8.25 \pm 5.44$ & $8.49 \pm 6.24$ & $8.03 \pm 4.55$ & 0.0761 \\
\hline $\mathrm{HbA} 1 \mathrm{c}[\%]$ & $5.46 \pm 0.31$ & $5.47 \pm 0.31$ & $5.44 \pm 0.31$ & 0.0280 \\
\hline $\mathrm{HbA} 1 \mathrm{c}[\mathrm{mmol} / \mathrm{mol}]$ & $36.2 \pm 3.4$ & $36.3 \pm 3.4$ & $35.0 \pm 3.4$ & 0.0280 \\
\hline Hyperglycemia, n & $368(19.7)$ & $232(25.7)$ & $136(14.1)$ & $<0.0001$ \\
\hline $\mathrm{TC}[\mathrm{mg} / \mathrm{dL}]$ & $199.9 \pm 41.3$ & $198.7 \pm 42.4$ & $200.9 \pm 40.15$ & 0.2425 \\
\hline $\mathrm{LDL}-\mathrm{C}[\mathrm{mg} / \mathrm{dL}]$ & $125.9 \pm 34.4$ & $125.9 \pm 34.7$ & $125.9 \pm 34.12$ & 0.9699 \\
\hline $\mathrm{HDL}-\mathrm{C}[\mathrm{mg} / \mathrm{dL}]$ & $50.3 \pm 13.1$ & $46.0 \pm 12.6$ & $54.3 \pm 12.35$ & $<0.0001$ \\
\hline TG [mg/dL] & $120.4 \pm 80.8$ & $138.2 \pm 99.5$ & $103.8 \pm 53.24$ & $<0.0001$ \\
\hline CRP $[\mathrm{mg} / \mathrm{dL}]$ & $2.77 \pm 5.63$ & $3.02 \pm 7.05$ & $2.54 \pm 3.86$ & 0.0735 \\
\hline Plasma creatinine $[\mathrm{mg} / \mathrm{dL}]$ & $0.82 \pm 0.17$ & $0.9 \pm 0.15$ & $0.75 \pm 0.15$ & $<0.0001$ \\
\hline$A C R[\mathrm{mg} / \mathrm{g}]$ & $15.67 \pm 187.88$ & $12.74 \pm 65.39$ & $18.39 \pm 253.42$ & 0.5028 \\
\hline $\log A C R[\mathrm{mg} / \mathrm{g}]$ & $0.78 \pm 0.35$ & $0.72 \pm 0.36$ & $0.84 \pm 0.33$ & $<0.0001$ \\
\hline Current smoking, $\mathrm{n}$ & $516(27.6)$ & $281(31.2)$ & $235(24.3)$ & 0.0011 \\
\hline
\end{tabular}

BMI - body mass index; SBP - mean systolic blood pressure; DBP - mean diastolic blood pressure; TC - total cholesterol; LDL-C - low-density lipoprotein cholesterol; HDL-C - high-density lipoprotein cholesterol; TG - triglycerides; ACR - albumin/creatinine ratio; WC - waist circumference; CRP - C-reactive protein; SD - standard deviation. 


\section{Results}

Characteristics of the population studied are presented in Table 1. Mean HbA1c level value was $5.46 \pm 0.31 \%$ $(36.2 \pm 3.4 \mathrm{mmol} / \mathrm{mol})$ and was significantly higher in men than in women (Table 1). HbA1c level was higher in subjects with hypertension $5.61 \pm 0.31 \%$ vs $5.39 \pm 0.29 \%$ $(37.8 \pm 3.4 \mathrm{mmol} / \mathrm{mol}$ vs $35.4 \pm 3.2 \mathrm{mmol} / \mathrm{mol}, \mathrm{p}<0.001)$ than without; higher in fasting hyperglycemia $-5.69 \pm 0.32 \%$ $(38.7 \pm 3.5 \mathrm{mmol} / \mathrm{mol})$ than in subjects with normal fasting glucose $-5.40 \pm 0.28 \%(35.5 \pm 3.1 \mathrm{mmol} / \mathrm{mol}, \mathrm{p}<0.001)$; and higher in subjects with abdominal obesity than without $-5.54 \pm 0.31 \%$ vs $5.34 \pm 0.27 \%(37.1 \pm 3.4 \mathrm{mmol} / \mathrm{mol}$ vs $34.9 \pm 3.0 \mathrm{mmol} / \mathrm{mol}, \mathrm{p}<0.001)$. Significant differences in HbA1c were observed in subjects with normal/low BMI, overweight and obesity: $5.34 \pm 0.26 \%$ vs $5.50 \pm 0.30 \%$ vs $5.62 \pm 0.31 \%$, respectively $(34.9 \pm 2.8 \mathrm{mmol} / \mathrm{mol}$ vs 36.6 $\pm 3.3 \mathrm{mmol} / \mathrm{mol}$ vs $37.93 .4 \mathrm{mmol} / \mathrm{mol}, \mathrm{p}<0.001)$. No significant difference in HbA1c was observed in smokers vs non-smokers.

Univariate analysis revealed numerous significant correlations of $\mathrm{HbA1c}$ with other covariants including age $(r=0.55)$ (Fig. 1), fasting glucose $(r=0.43)$, WC $(r=0.36)$, $\operatorname{BMI}(r=0.36)$, SBP $(r=0.28)$, LDL-C $(r=0.26)$, total cholesterol $(r=0.23)$, and DBP $(r=0.21)$. Correlation coefficients for insulin, creatinine, ACR, triglycerides, and HDL-C, although significant, were below 0.2 .

The best final multivariate linear regression model was selected with all subsets regression procedures and included following variables independently associated with HbA1c levels: age, FPG, BMI, TC, HDL-C, ACR, TG, and gender (Table 2). Albumin-creatinine ratio and gender were not significant correlates. The final model explained $40.25 \%$ of HbA1c variance. The most important covariant of $\mathrm{HbA1c}$, irrespective of the method of assessment of the relative importance of variables selected to the final model, was age, followed by FPG and, in the majority of methods, BMI (Fig. 2). Relative $\mathrm{R}^{2}$ for the other covariants was negligible, below $10 \%$.

Table 2. Multivariate associations between $\mathrm{HbA1c}$ and clinical/ biochemical parameters in the final model expressed as adjusted beta coefficients

\begin{tabular}{|l|c|c|}
\hline \multicolumn{1}{|c|}{ Parameter } & beta & p-value \\
\hline Age & 0.40 & $<0.001$ \\
\hline Gender & -0.02 & ns \\
\hline FPG & 0.26 & $<0.001$ \\
\hline BMI & 0.09 & $<0.001$ \\
\hline TC & 0.10 & $<0.001$ \\
\hline HDL-C & -0.13 & $<0.001$ \\
\hline TG & -0.05 & $<0.05$ \\
\hline logACR & 0.02 & ns \\
\hline
\end{tabular}

FPG - fasting plasma glucose; BMI - body mass index; TC - total cholesterol; HDL-C - HDL cholesterol; TG - triglycerides; ACR - albumin/ creatinine ratio; ns - not significant; significant $\mathrm{p}$-value $<0.05$.

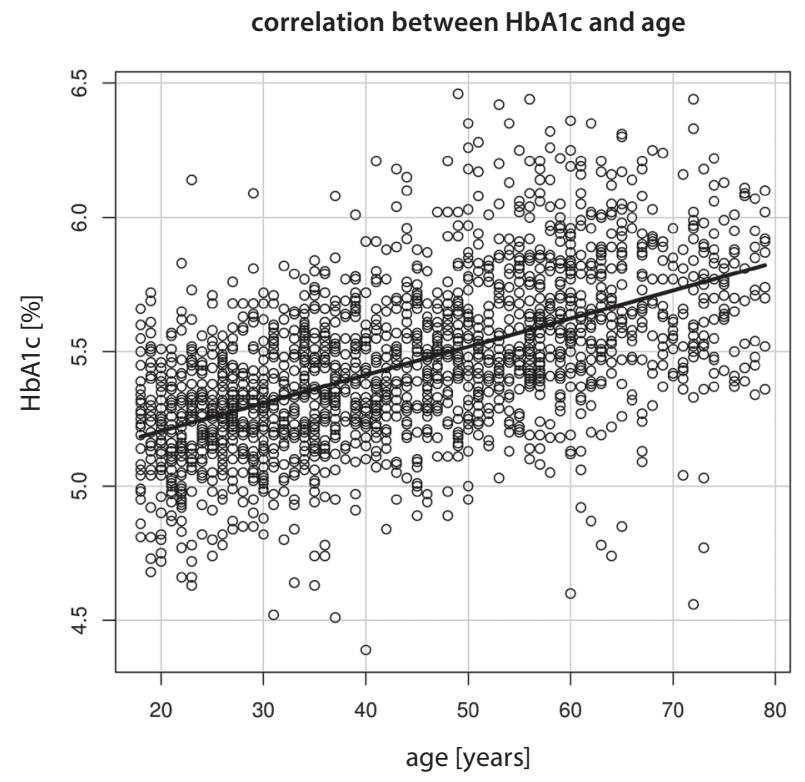

Fig. 1. Correlation between $\mathrm{HbA1c}$ and age

\section{Discussion}

Our study revealed that the mean HbA1c level in a representative sample of the Polish population without previously diagnosed diabetes and with non-diabetic range of HbA1c was similar to other non-diabetic population studies. ${ }^{6,11} \mathrm{HbA1c}$ levels were higher in subjects with certain well-known CV risk factors, namely male gender, obesity, abdominal obesity, hypertension, and hypercholesterolemia but not in current smokers.

Univariate analysis revealed numerous significant correlations with other covariants, with highest $r$ values found for age and also for FPG, WC and BMI. It should be noted that the correlation between FPG and HbA1c was not very high and similar to that found in the Dutch general population. ${ }^{12}$ In the large Finnish METSIM study, the respective r coefficient in non-diabetic men was even lower - 0.207. On the other hand, our analysis, when compared with METSIM study, revealed a 2-fold higher $\mathrm{r}$ coefficients for HbA1c levels and age, BMI and SBP. ${ }^{11}$

In our study, age, glucose, BMI, total cholesterol, HDL-C, and TG were significant and independent determinants of HbA1c level. In contrast to other studies, other covariants including $\mathrm{CRP}^{11}$ and smoking ${ }^{6}$ were not included into the model. The main determinant of HbA1c variance in our participants was age, which explained half of the variance of HbA1c in the model, similarly to the METISM study. The relative $\mathrm{R}^{2}$ determined by FPG was approx. $25 \%$, as reported in METSIM study (24.7\%). ${ }^{11}$

In METSIM study, age, FPG, CRP, genetic risk score, and smoking were the most important determinants of the variance in HbA1c among participants without DM2, explaining $12-14 \%$ of variance in HbA1c, whereas insulin secretion and insulin sensitivity indices explained only $<2 \%$ of variance. ${ }^{11}$ In a Dutch study performed in non-diabetic 


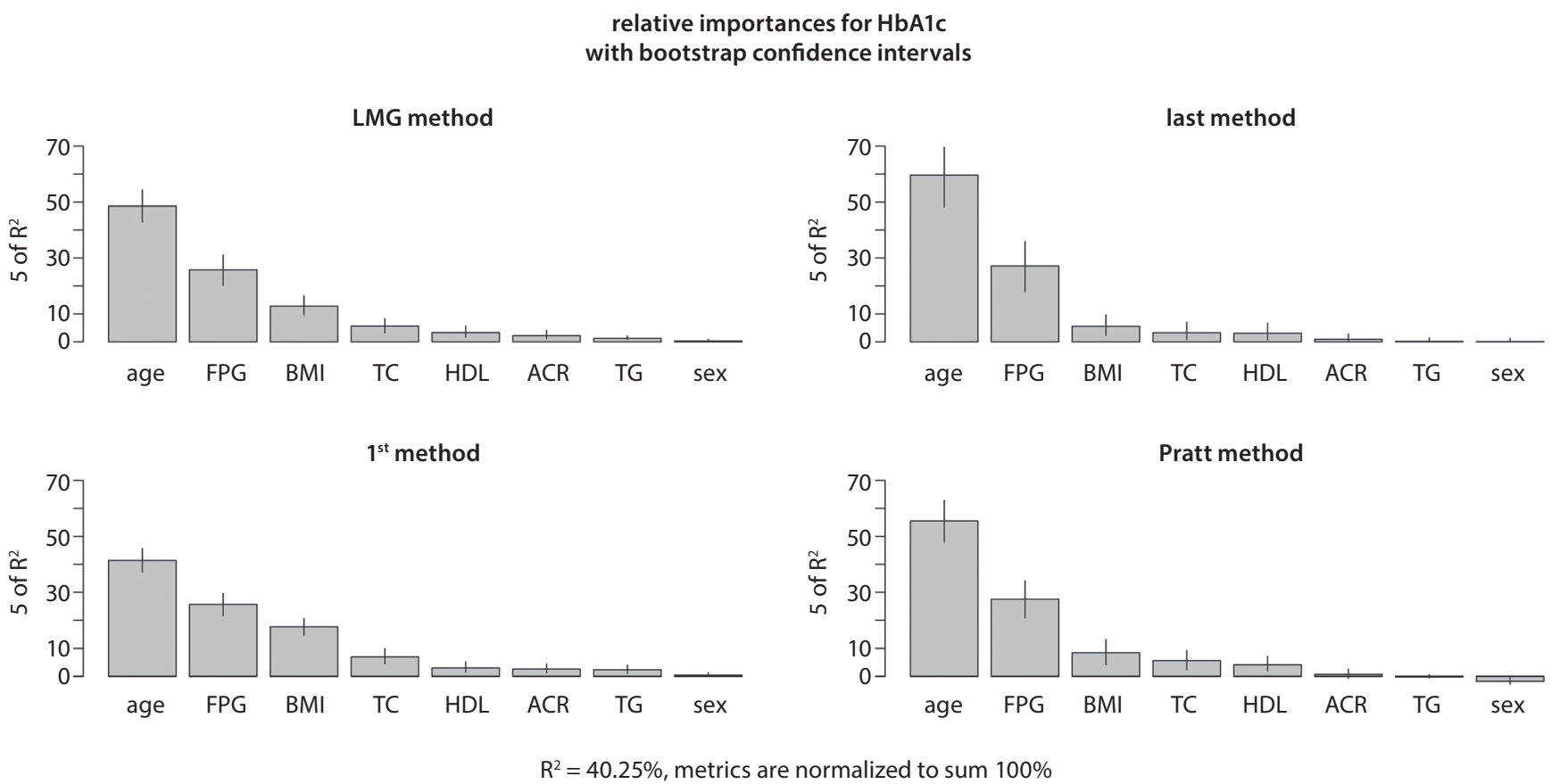

Fig. 2. Relative importance for $\mathrm{HbA1c}$ expressed as $\%$ of $\mathrm{R}^{2}$ for the final model calculated using different methods

adults, regression model that included age, gender, BMI, mean corpuscular hemoglobin, mean corpuscular hemoglobin concentration, current smoking, and alcohol consumption explained only $26 \%$ of HbAlc variance. ${ }^{6}$

Our model predicted HbA1c levels more precisely, explaining $40 \%$ of the variance of HbA1c compared with $14-26 \%$ of the variance in other studies. ${ }^{6,11}$ Differences between our and other studies may be explained by the lower mean age of our subjects, resulting in lower incidence of concomitant $\mathrm{CV}$ risk. Other studies have shown that variability in $\mathrm{HbA} 1 \mathrm{c}$ may be contributed to age, ethnicity, smoking, anemia, and genetic factors. ${ }^{13-16}$ Apart from age and, to a minor extent FPG and BMI, the influence of other variables in our model was small, almost negligible.

Of note, multivariate regression models including traditional and non-traditional risk factors/markers explain only $40 \%$ of variance of HbA1c. Therefore, the physiological link between HbA1c and CV risk in non-diabetic subjects remains unexplained, thus suggesting that HbA1c may be an independent CV risk factor. Prediabetes is a wellknown CV risk factor and HbA1c should be considered a useful independent CV risk factor also in the diabetesfree population.

The studies on the possible determinants of HbA1c levels in non-diabetic populations are difficult to compare due to methodological differences, including diagnosis of diabetes, selection of the potential covariates and statistical methodology.

Several limitations of our study must be mentioned. We did not perform oral glucose tolerance test (OGTT) and the participants had only 1 FPG measurements; therefore, to minimize the possibility of including patients with DM to the analysis, we excluded the participants with HbA1c $\geq 6.5 \%$ as in other studies. ${ }^{17}$ However, post-load glucose measurement did not improve the prediction of HbA1c. ${ }^{11}$

\section{Conclusions}

HbA1c in non-diabetic level is associated with some CV risk factors, mainly with age. Since known risk factors explain less than half of its variance, the inclusion of HbA1c into risk assessment may increase the performance of algorithms predicting $\mathrm{CV}$ risk.

\section{ORCID iDs}

Bartosz Symonides (1) https://orcid.org/0000-0002-5933-609X Bogdan Solnica (1) https://orcid.org/0000-0002-0121-8154 Grzegorz Placha (1) https://orcid.org/0000-0002-6106-2454 Ewa Pędzich-Placha (1) https://orcid.org/0000-0001-8965-0736 Marcin Rutkowski (1) https://orcid.org/0000-0002-4985-2250 Piotr Bandosz (1) https://orcid.org/0000-0002-6395-6216 Zbigniew Gaciong (1) https://orcid.org/0000-0002-6666-3734 Tomasz Zdrojewski @i] https://orcid.org/0000-0001-6015-8561

\section{References}

1. Pradhan AD, Rifai N, Buring JE, Ridker PM. Hemoglobin A1c predicts diabetes but not cardiovascular disease in nondiabetic women. Am J Med. 2007;120(8):720-727.

2. Selvin E, Steffes MW, Zhu H, et al. Glycated hemoglobin, diabetes, and cardiovascular risk in nondiabetic adults. N Engl J Med. 2010; 362(9):800-811.

3. Khaw K-T, Wareham N, Bingham S, Luben R, Welch A, Day N. Association of hemoglobin A1c with cardiovascular disease and mortality in adults: The European prospective investigation into cancer in Norfolk. Ann Intern Med. 2004;141(6):413-420. 
4. Seino Y, Nanjo K, Tajima N, et al. Report of Committee on the Classification and Diagnostic Criteria of Diabetes Mellitus. J Diabetes Investig. 2010;1:(5)212-228.

5. Matsushita K, Blecker S, Pazin-Filho A, et al. The association of hemoglobin a1c with incident heart failure among people without diabetes: The atherosclerosis risk in communities study. Diabetes. 2010; 59(8):2020-2026.

6. Jansen H, Stolk RP, Nolte IM, Kema IP, Wolffenbuttel BHR, Snieder H. Determinants of HbA1c in nondiabetic Dutch adults: Genetic loci and clinical and lifestyle parameters, and their interactions in the Lifelines Cohort Study. J Intern Med. 2013;273(3):283-293.

7. Sarwar N, Aspelund T, Eiriksdottir G, et al. Markers of dysglycaemia and risk of coronary heart disease in people without diabetes: Reykjavik prospective study and systematic review. PLoS Med. 2010; 7(5):e1000278.

8. Gerstein $\mathrm{HC}$, Islam S, Anand S, et al. Dysglycaemia and the risk of acute myocardial infarction in multiple ethnic groups: An analysis of 15,780 patients from the INTERHEART study. Diabetologia. 2010;53(12):2509-2517.

9. Preiss D, Sattar N. HbA1c: A useful cardiovascular risk marker in those without diabetes? Diabetologia. 2010;53(12):2468-2469.

10. Rutkowski $M$, Bandosz $P, C$ zupryniak $L$, et al. Prevalence of diabetes and impaired fasting glucose in Poland: The NATPOL 2011 Study. Diabet Med. 2014;31(12):1568-1571.
11. Fizelova M, Stancakova A, Lorenzo C, et al. Glycated hemoglobin levels are mostly dependent on nonglycemic parameters in 9398 Finnish men without diabetes. J Clin Endocrinol Metab. 2015;100(5): 1989-1996.

12. van 't Riet $E$, Alssema M, Rijkelijkhuizen JM, Kostense PJ, Nijpels G, Dekker JM. Relationship between A1C and glucose levels in the general Dutch population: The new Hoorn study. Diabetes Care. 2010;33(1):61-66.

13. Sacks DB, Bruns DE, Goldstein DE, Maclaren NK, McDonald JM, Parrott $M$. Guidelines and recommendations for laboratory analysis in the diagnosis and management of diabetes mellitus. Clin Chem. 2002;48(3):436-472.

14. Pani LN, Korenda L, Meigs JB, et al. Effect of aging on A1C levels in individuals without diabetes: Evidence from the Framingham Offspring Study and the National Health and Nutrition Examination Survey 2001-2004. Diabetes Care. 2008;31(10):1991-1996.

15. Soranzo N, Sanna S, Wheeler E, et al. Common variants at 10 genomic loci influence hemoglobin A1(c) levels via glycemic and nonglycemic pathways. Diabetes. 2010;59(12):3229-3239.

16. Davidson MB, Schriger DL. Effect of age and race/ethnicity on $\mathrm{HbA1c}$ levels in people without known diabetes mellitus: Implications for the diagnosis of diabetes. Diabetes Res Clin Pract. 2010;87(3): 415-421.

17. Haring $R$, Baumeister SE, Lieb $W$, et al. Glycated hemoglobin as a marker of subclinical atherosclerosis and cardiac remodeling among non-diabetic adults from the general population. Diabetes Res Clin Pract. 2014;105(3):416-423. 